\title{
Controllable synthesis of hollow porous silica nanotubes/CuS nanoplatform for targeted chemo-photothermal therapy
}

\author{
Shanshan Huang ${ }^{1^{*}}$, Ping'an $\mathrm{Ma}^{2}$, Yi Wei ${ }^{2}$, Ziyong Cheng ${ }^{2 *}$, Bei Liu $^{2}$, Xiaoran Deng ${ }^{2}$, Zhongxi Xie ${ }^{2}$, \\ Bengang Xing ${ }^{4}$ and Jun Lin ${ }^{2,3^{*}}$
}

\begin{abstract}
The design and synthesis of multifunctional nanocarriers for efficient synergistic cancer therapy have drawn great research interests in recent years. In this work, a nanoplatform for chemo-photothermal therapy with targeting ligand was developed. Hollow porous structured silica nanotubes (SNTs) with controllable lengths decorated with $\mathrm{CuS}$ nanoparticles (NPs) on the surface as photothermal agents were prepared and further conjugated with lactobionic acid groups as a cancer cell target. SNTs with average lengths of 40, 55 and $150 \mathrm{~nm}$ were obtained and further functionalized as drug carriers. The smallest bifunctional SNTs with targeting groups show good biocompatibility and highest cellular uptake for HepG2 cells. The release of doxorubicin hydrochloride (DOX) from the SNTs was dependent on the $\mathrm{pH}$ of the buffer solution and 808-nm near infrared (NIR) light irradiation. The integration of photothermal therapy (PTT) of CuS NPs and chemotherapy of anticancer drug leads to a better tumor inhibition effect than the individual therapy alone in vitro and in vivo. These results demonstrate potential applications of the nanocomposites as vector for efficient chemo-photothermal therapy.
\end{abstract}

Keywords: multifunctional nanocarriers, chemo-photothermal therapy, silica nanotubes, CuS nanoparticles, cancer cell target

\section{INTRODUCTION}

Mesoporous silica-based nanomaterials have been widely investigated in biomedical areas for their large pore volume, high surface area, chemically modifiable surfaces, and excellent biocompatibility during the last decades [1-
3]. Although different polymeric nanosystems have demonstrated a great potential for cancer therapy, the advantages of silica nanoparticles (NPs) endow them with promising future for therapeutics [4]. Mesoporous silica shells growing on the surface of functional inorganic nanoparticle cores through hard-templating routes provide a novel strategy for constructing the nanomedicine system [5]. $\mathrm{Fe}_{3} \mathrm{O}_{4} @ \mathrm{MSN}$ (heterogeneous rattle-type $\mathrm{Fe}_{3} \mathrm{O}_{4} @ \mathrm{mSiO}_{2}$ ) which utilize the mesoporous silica coating and functional core were prepared for drug delivery $[6,7]$. Previous studies demonstrated that rod-like multifunctional MSNs had been extensively applied in cancer cell metastasis, drug/DNA delivery and cell imaging [810]. Silica nanotubes (SNTs) with different aspect ratios were synthesized using a template of rod-like nickel hydrazine complex NPs [11,12]. Furthermore, a multifunctional drug delivery system comprising hollow silica tubes, a superparamagnetic magnetite nanoparticle deposition and a hyaluronic acid decorating was prepared for cancer-targeting drug delivery [13]. Yang et al. [14] synthesized hollow-structured mesoporous silica rods (HMSRs) with controllable aspect ratios. The endocytosis of HMSR to HeLa cells is shape-dependent and the HMSRs with higher length values show more efficient internalization than those shorter ones.

Photothermal therapy (PTT) has attracted great attention owing to its non-invasive therapy for utilizing photothermal transduction agents (PTAs) to convert light to generate heat energy for tumor ablation [15-17]. Copper sulfide (CuS) NPs have been greatly concerned as a

\footnotetext{
${ }^{1}$ College of Chemistry and Pharmaceutical Engineering, Huanghuai University, Zhumadian 463000, China

${ }^{2}$ State Key Laboratory of Rare Earth Resource Utilization, Changchun Institute of Applied Chemistry, Chinese Academy of Sciences, Changchun 130022, China

${ }^{3}$ School of Applied Physics and Materials, Wuyi University, Jiangmen 529020, China

${ }^{4}$ School of Physical \& Mathematical Sciences, Nanyang Technological University, Singapore 637371, Singapore

* Corresponding authors (emails: sshuang@huanghuai.edu.cn (Huang S); zycheng@ciac.ac.cn (Cheng Z); jlin@ciac.ac.cn (Lin J))
} 
prospective inorganic PTA for PTT because of their low cost, good biocompatibility and biodegradable properties [18-20]. Multifunctional nanocomposites based on CuS have been designed and utilized for cancer therapy [2123]. $\mathrm{NaLnF}_{4}: \mathrm{Yb} / \mathrm{Er} @ \mathrm{Cu}_{2-x} \mathrm{~S}(\mathrm{Ln}=\mathrm{Y}, \mathrm{Yb}, \mathrm{Lu})$ hybrid nanoprobes with a core-satellite structure were synthesized for NIR-II optical imaging, small tumor detection and PTT [24,25]. CuS-decorated mesoporous silica NPs (MSNs) as drug delivery systems for tumor therapy were reported previously. A PEG-modified MSN@CuS nanocomposite was developed for the synergistic therapy of photothermal and chemotherapy with obvious cytotoxicity to hepatocellular carcinoma cells in vitro [26]. Compared with normal MSN, hollow mesoporous silica nanospheres have attracted more attention as the hollow voids provide more space for the loading of drug molecules $[4,27,28]$. Hollow mesoporous silica/CuS nanospheres conjugated with folic acid were designed for the chemo-photothermal targeting therapy of HeLa cells with 980-nm laser irradiation [29]. However, the in vivo tumor inhibition effect on mice for these composites was not demonstrated. In addition, $980 \mathrm{~nm}$ irradiation could induce overheating effect and induce tissue damage [30,31]. 808-nm laser irradiation for PTT can not only alleviate the overheating caused by the $980 \mathrm{~nm}$ laser, but also cause higher tissue penetration depth because of the minimum absorbance for biological tissues [32,33]. Smaller particles are considered to be beneficial to the therapy because of the efficient cellular uptake and long blood circulation time [2,34]. Therefore, design and synthesis of NPs with relatively small particle size for the combination of PTT and chemotherapy are highly desired.

In this work, hollow SNTs with average lengths of 40, 55 and $150 \mathrm{~nm}$ were prepared by using the nickelhydrazine complex as template followed by an etching process with acid and hot water. After functionalization with amino and lactobionic acid (LA) groups, these SNTs were modified with CuS NPs through electrostatic adsorption. LA bonding with the surface of the silica shell could efficiently target to hepatoma cells which overexpress the asialoglycoprotein receptor [35,36]. The permeable silica layer and hollow cavity of the nanotubes provide space for the loading of doxorubicin (DOX), a widely used chemotherapeutic drug. Furthermore, the size effects of these functionalized SNTs on the endocytosis and biocompatibilities were investigated. The antitumor effect of the controllable SNTs with LA groups targeting for the hepatoma cells was studied. The nanocomposite with combination of PTT, chemotherapy and targeting group could be a synergistic platform for cancer treatment.

\section{EXPERIMENTAL SECTION}

\section{Materials}

Polyoxyethylene (20) cetyl ether (Brij 58) was purchased from Sigma-Aldrich. Hydrazine hydrate, tetraethyl orthosilicate (TEOS), nickel chloride hexahydrate $\left(\mathrm{NiCl}_{2} \cdot 6 \mathrm{H}_{2} \mathrm{O}\right)$, diethylamine, sodium citrate, sodium hydroxide $(\mathrm{NaOH})$, hydrochloric acid $(\mathrm{HCl}, 37.5 \%)$, cyclohexane, isopropanol, and dimethyl sulfoxide (DMSO) were purchased from Beijing Chemical Cooperation Ltd. $\mathrm{N}$-(3-dimethylaminopropyl)- $\mathrm{N}$-ethylcarbodiimide hydrochloride (EDC, 98\%), N-hydroxysuccinimide (NHS, 98\%), (3-aminopropyl)triethoxysilane (APTES, $\geq 98 \%$ ), fluorescein isothiocyanate (FITC), 4',6-diamidino-2-phenylindole (DAPI) and methoxy-PEG-thiol (SH-PEG, molecular weight $1000 \mathrm{Da}$ ) were purchased from SigmaAldrich. $\mathrm{Na}_{2} \mathrm{~S} \cdot 9 \mathrm{H}_{2} \mathrm{O}, \mathrm{CuCl}_{2} \cdot 2 \mathrm{H}_{2} \mathrm{O}$, and LA were purchased from Sinopharm Chemical Reagent. DOX hydrochloride was purchased from Nanjing Duobo Chemical Limited Company. All chemicals were used as received without further purification.

\section{Synthesis of hollow porous SNTs}

The hollow porous structured SNTs were fabricated according to the previous reports with modifications [11]. Brij58 (17 g) was dispersed in $30 \mathrm{~mL}$ of cyclohexane and then stirred at $55^{\circ} \mathrm{C}$ in water bath. One milliliter of $\mathrm{NiCl}_{2}$ solution $\left(0.8 \mathrm{~mol} \mathrm{~L}^{-1}\right)$ was added and mixed to reach complete dissolution. Then, $1.0 \mathrm{~mL}$ of hydrazine hydrate was added drop by drop. Two milliliter of diethylamine and $5.0 \mathrm{~mL}$ of TEOS were added into the above system after continuous stirring for $3 \mathrm{~h}$. The sample was then centrifuged and washed with isopropanol and water. The products were dispersed in $100 \mathrm{~mL}$ of $\mathrm{HCl}$ solution $\left(2 \mathrm{~mol} \mathrm{~L}^{-1}\right)$ under sonication and stirred for $2 \mathrm{~h}$. After centrifugation of the suspension and being washed with deionized water, the final SNTs with a length of $40 \mathrm{~nm}$ were collected. SNTs with different lengths of 55 and $150 \mathrm{~nm}$ were also prepared by tuning the volume of $\mathrm{NiCl}_{2}$ solution to 1.2 and $2.0 \mathrm{~mL}$, respectively. In order to improve the porous structures of these SNTs, $100 \mathrm{mg}$ of the as-prepared SNTs were sonicated in deionized water at $70^{\circ} \mathrm{C}$ and stirred for $1 \mathrm{~h}$. After centrifugation and being dried in the oven, the products were finally obtained.

\section{Synthesis of SNT-NH}

The SNTs (100 mg) were dispersed in $50 \mathrm{~mL}$ of ethanol under sonication. Deionized water $(250 \mu \mathrm{L})$ and APTES 
$(250 \mu \mathrm{L})$ were added and the mixture was stirred in $45^{\circ} \mathrm{C}$ water bath for $8 \mathrm{~h}$. The products (denoted as SNT- $\mathrm{NH}_{2}$ ) were centrifuged, washed and dried at $60^{\circ} \mathrm{C}$ for further use.

\section{Coupling of lactobionic acid onto SNT- $\mathrm{NH}_{2}$} The suspension of $50 \mathrm{mg}$ of SNT- $\mathrm{NH}_{2}$ in $1 \mathrm{~mL}$ of DMSO was sonicated for $10 \mathrm{~min} .35 .8 \mathrm{mg}$ of LA, $31.0 \mathrm{mg}$ of EDC and $11.5 \mathrm{mg}$ of NHS were dissolved completely in $3 \mathrm{~mL}$ of DMSO. Then the mixture was added into the dispersion of SNT- $\mathrm{NH}_{2}$. The reaction was conducted for $72 \mathrm{~h}$ at room temperature. The product (denoted as SNT-LA) was washed by DMSO and water, and dried for later use.

\section{Synthesis of CuS NPs and SNT-LA-CuS-PEG nanocomposites}

The CuS NPs in water were prepared based on a previously reported method [26]. A dark-green solution of $\mathrm{CuS}$ NPs with coating of citrate was obtained and stored for later use. SNT-LA-CuS was synthesized through the electrostatic interaction between the protonated $\mathrm{NH}_{2}$ and negative CuS. $10 \mathrm{mg}$ of the SNT-LA was dispersed in $2 \mathrm{~mL}$ of deionized water under sonication. $7 \mathrm{~mL}$ of the prepared $\mathrm{CuS}$ was introduced into the above mixture and stirred for $1 \mathrm{~h}$. Then the sample was centrifuged, washed and dispersed in $10 \mathrm{~mL}$ of deionized water. Then, SHPEG (6 mg) was added and the suspension was stirred for $8 \mathrm{~h}$ at room temperature to tether PEG onto the outer surface of the CuS NPs. The SNT-LA-CuS-PEG nanocomposite was obtained after centrifugation and washing. SNT- $\mathrm{NH}_{2}$-CuS-PEG was prepared by using the similar method.

\section{Synthesis of FITC labeled SNT}

To fabricate SNT-FITC, $6 \mu \mathrm{L}$ of APTES was added into $2 \mathrm{~mL}$ of DMSO containing $2.0 \mathrm{mg}$ of FITC. The reaction was carried out in the dark for $12 \mathrm{~h} .25 \mathrm{mg}$ of SNT-LA dispersed in $20 \mathrm{~mL}$ of ethanol was added into the above mixture and refluxed at $80^{\circ} \mathrm{C}$ for another $10 \mathrm{~h}$. The sample was centrifuged, washed with ethanol and dialyzed against deionized water for $72 \mathrm{~h}$. The final sample was collected and dried for further use. Then the obtained product was functionalized with CuS and PEG by the method mentioned above.

\section{Measurement of photothermal performance}

Dispersions of the SNT-LA-CuS-PEG nanocomposite at different concentrations $\left(0,50,100,200,400 \mu \mathrm{g} \mathrm{mL}^{-1}\right)$ were irradiated by $808-\mathrm{nm}$ an laser (powder density,
$2.0 \mathrm{~W} \mathrm{~cm}^{-2}$ ). The temperature of the solutions was monitored by a digital thermometer by using a thermocouple probe. Deionized water was used as control.

\section{In vivo photothermal imaging of the NPs}

Healthy female BALB/C mice (about $20 \mathrm{~g}$ ) were purchased from Changchun Institute of Biological Products. All the animal experiments were in accordance with the criteria of the National Regulation of China for the Care and Use of Laboratory Animals. Firstly, H22 cells (murine hepatocarcinoma cell line) were injected in the left axilla of each female mouse six days earlier to establish the tumor model. Balb/c mice bearing H22 tumors were intravenously injected with $100 \mu \mathrm{L}$ of NPs $\left(20 \mathrm{mg} \mathrm{kg}^{-1}\right.$ per each mouse). The thermal images at different time points after irradiation were provided by using an FLIR infrared camera when tumors were exposed to the 808-nm laser.

\section{DOX loading and release}

The typical process for DOX loading was as follows: first, $5 \mathrm{mg}$ of SNT-LA-CuS-PEG was dispersed in an aqueous solution of DOX $\left(1 \mathrm{~mL}, 2.5 \mathrm{mg} \mathrm{mL}^{-1}\right)$, and shaken at $37^{\circ} \mathrm{C}$ for $24 \mathrm{~h}$ to reach the equilibrium state. The DOX loaded SNT-LA-CuS-PEG was obtained by centrifugation. The supernatant solution containing the free DOX was measured by a UV-vis absorption spectrophotometer at $480 \mathrm{~nm}$ to calculate the amount of DOX loaded in the nanocomposites. The loading content of DOX was calculated by the following equation: drug loading content $(\%)=100 \times($ weight of DOX in the SNT-LA-CuSPEG) / (weight of the SNT-LA-CuS-PEG + weight of DOX loaded into the SNT-LA-CuS-PEG). The DOX release from SNT-LA-CuS-PEG/DOX with or without the 808-nm NIR laser irradiation was carried out by dispersing the DOX loaded samples in $2.0 \mathrm{~mL}$ of buffer solution at $\mathrm{pH} 7.4$ and 5.0 under shaking, respectively. At the predetermined time points, the suspensions were centrifuged and the supernatant was collected for analysis and an equal volume of fresh medium was added. In the presence of laser irradiation, the dispersion of SNT-LACuS-PEG/DOX was irradiated by an 808-nm NIR laser $\left(2.0 \mathrm{~W} \mathrm{~cm}^{-2}\right)$ for $5 \mathrm{~min}$ every hour. The sustained release of DOX in the buffer solution at different time points was monitored with a UV-vis spectrophotometer at $480 \mathrm{~nm}$.

\section{In vitro cytotoxicity of the SNT-LA-CuS-PEG NPs}

About 8000 L929 fibroblast cells were cultured in $100 \mu \mathrm{L}$ of Dulbecco's modified Eagle medium (DMEM) supplemented with $1 \%(v / v)$ penicillin, $1 \%(v / v)$ streptomycin and $10 \%(w / v)$ fetal bovine serum at $37^{\circ} \mathrm{C}$ under a hu- 
midified $5 \% \mathrm{CO}_{2}$ atmosphere in a 96-well plate. Then $100 \mu \mathrm{L}$ of the solutions with NP concentrations of 6.25 , $12.5,25,50,100$ and $200 \mu \mathrm{g} \mathrm{mL}^{-1}$ were added to the cells, respectively. Untreated cells were used as control. The cells were incubated at $37^{\circ} \mathrm{C}$ in $5 \% \mathrm{CO}_{2}$ for $24 \mathrm{~h}$. After that, $20 \mu \mathrm{L}$ of 3 -[4,5-dimethylthiazol-2-yl]-2,5-diphenyltetrazolium bromide (MTT) solution (dispersed in DMEM with $10 \%$ concentration) was added into each well with continued cultivation for $4 \mathrm{~h}$. After the supernatant was removed, $150 \mu \mathrm{L}$ of DMSO was added to each well. Finally, the absorbance at $490 \mathrm{~nm}$ of the plate was recorded using a microplate reader (Thermo Multiskan MK3).

\section{Cellular uptake assay}

For quantification of the cell uptake of the samples, HepG2 cells were cultured in 6-well plates at a density of $2 \times 10^{5}$ cells per well for $24 \mathrm{~h}$. These cells were then cultured with SNT-NH${ }_{2}$-CuS-PEG $(100 \mu \mathrm{g})$ or SNT-LACuS-PEG $(100 \mu \mathrm{g})$ at $37^{\circ} \mathrm{C}$ for 1,4 and $6 \mathrm{~h}$. After being washed with phosphate buffer saline (PBS) three times, the cells were lysed by the cell lysis buffer. Inductively coupled plasma-optical emission spectrometer (ICP-OES) (iCAP 6300 of Thermo Scientific) was used to measure the $\mathrm{Si}$ content in the cell lysis solution.

\section{CLSM images}

For the cell uptake experiment, HepG2 cells were plated in 6-well plates at a density of $1 \times 10^{5}$ cells per well and allowed to attach and grow for $24 \mathrm{~h}$. These cells were then incubated with SNT-40-NH2-CuS-PEG-FITC, SNT-40LA-CuS-PEG-FITC, SNT-55-LA-CuS-PEG-FITC, SNT150-LA-CuS-PEG-FITC and DAPI at $37^{\circ} \mathrm{C}$ for 1 and $6 \mathrm{~h}$, respectively. After that, the cells were washed with $\mathrm{PBS}$ three times to remove any residual NPs. $2.5 \%$ glutaraldehyde ( $1 \mathrm{~mL}$ per well) was added and the cells were cultured at $37^{\circ} \mathrm{C}$ for another $10 \mathrm{~min}$. The samples were visualized by confocal laser scanning microscopy (CLSM, Leica TCS SP8).

\section{Chem-photothermal therapy}

HepG2 cells were cultured in 96-well plates in a complete medium for $24 \mathrm{~h}$. Then, the SNT-LA-CuS-PEG/DOX dispersed in DMEM was added into the wells. After $6 \mathrm{~h}$ of incubation, the cells were washed with PBS and replaced with fresh medium. For the combined therapy, the cells were irradiated with an $808-\mathrm{nm}$ laser $\left(2.0 \mathrm{~W} \mathrm{~cm}^{-2}\right)$ for $5 \mathrm{~min}$ and further incubated for $24 \mathrm{~h}$ in the dark. The cell viability after integrated therapy was evaluated by the standard MTT assay.

\section{In vivo photothermal/chemotherapy anticancer efficacy}

The tumor-bearing BALB/C mice model was established as described above. The tumors were allowed to grow to a size around $60-100 \mathrm{~mm}^{3}$, then the tumor-bearing mice were divided into five groups ( $n=6$, each group), group 1 : control; group 2: DOX only; group 3: SNT-LA-CuS-PEG (NPs)/DOX; group 4: NPs+NIR irradiation; group 5: NPs/DOX+NIR irradiation. The DOX, NPs and (NPs)/ DOX were injected intratumorally with $4.0 \mathrm{mg}$ DOX per $\mathrm{kg}$ body weight on day 1 and 4, respectively. After $12 \mathrm{~h}$, the tumors in the groups of NPs+NIR irradiation and $\mathrm{NPs} / \mathrm{DOX}+\mathrm{NIR}$ irradiation were exposed to an 808-nm laser with an output power density of $2.0 \mathrm{~W} \mathrm{~cm}^{-2}$ for $15 \mathrm{~min}$ ( $5 \mathrm{~min}$ break after $5 \mathrm{~min}$ irradiation). The tumor sizes of the mice were determined by using a digital caliper every two days and body weights were measured at the same time. The tumor volume was calculated with the formula: $V=L W^{2} / 2$, where $L$ and $W$ refer to the length $(\mathrm{mm})$ and width $(\mathrm{mm})$ of the tumor, respectively.

\section{Histological examination}

To determine the histological changes of the main organs, one tumor-bearing mouse in each group was sacrificed at the 14th day after treatment. Afterward, the tumors and organs were collected, successively dehydrated and embedded in liquid paraffin. The sliced organ tissues (3-5 mm) were stained with hematoxylin and eosin (H\&E) and imaged by a microscope.

\section{Characterizations}

Powder X-ray diffraction (XRD) measurements were performed on a D8 Focus diffractometer (Bruker) with $\mathrm{Cu} \mathrm{Ka}$ radiation $(\lambda=0.15405 \mathrm{~nm})$. The X-ray photoelectron spectra (XPS) were taken on a VG ESCALAB MK II electron energy spectrometer using $\mathrm{Mg} \mathrm{Ka}$ $(1253.6 \mathrm{eV})$ as the X-ray excitation source. The (UV-vis)NIR adsorption spectra were recorded on Shimadzu UV3600 spectrophotometer. Fourier-transform infrared (FTIR) spectra were recorded on a Perkin-Elmer 580B IR spectrophotometer using the $\mathrm{KBr}$ pellet technique. Transmission electron microscopy (TEM) micrographs were obtained from an FEI Tecnai G2 S-Twin transmission electron microscope with a field emission gun operating at $200 \mathrm{kV}$. Zeta potential distribution measurements were recorded on a Zetasizer Nano ZS (Malvern Instruments Ltd, UK). MTT experiments were carried out by using a microplate reader (Thermo Multiskan MK3), while the instrument of UCLM was rebuilt on an inverted fluorescence microscope (Nikon Ti-S). The $\mathrm{N}_{2}$ adsorption-desorption isotherms of the samples 
were collected by the Micromeritics ASAP 2020 M apparatus. The surface area and the pore size distribution of the samples were respectively calculated by the Brunauer -Emmett-Teller (BET) and Barrett-Joyner-Halenda $(\mathrm{BJH})$ methods. All the measurements were performed at room temperature.

\section{RESULTS AND DISCUSSION}

The fabrication and application of the anticancer drug carrier combined with chemotherapy and photothermal therapy are shown in Scheme 1. Porous SNTs were prepared by using the rod like nickel-hydrazine complex template followed by the acid etching and hot water corrosion process. The SNTs were sequentially functionalized with amino groups and a part of them were further conjugated with LA on the surface of silica for the targeting of asialoglycoprotein receptor over expressing hepatoma cells. The residual $\mathrm{NH}_{2}$ could bind with citrate coated CuS NPs and form SNT-LA-CuS nanocomposites due to the electrostatic adsorption. CuS NPs can absorb NIR laser and generate heat to destroy the cancer cells. PEG-SH was further grafted to improve the dispersity of the NPs. The antitumor drug DOX was introduced into the space of the SNT-LA-CuS-PEG for the chemotherapy treatment.

The TEM image (Fig. 1a) demonstrates the monodispersed rod-like morphology of the SNT-40 sample with an average size of $40 \mathrm{~nm} \times 25 \mathrm{~nm}$ and shell thickness of $7 \mathrm{~nm}$. The SNTs with sizes of $55 \mathrm{~nm} \times 23 \mathrm{~nm}$ and $150 \mathrm{~nm} \times 20 \mathrm{~nm}$ were also fabricated by tuning the volume of the $\mathrm{NiCl}_{2}$ solution in the reaction, as shown in Figs S1, S2.

The pore structure of the hollow STNs was investigated through the $\mathrm{N}_{2}$ adsorption-desorption isotherm (Fig. 1c and Fig. S3). The pristine SNTs showed a hysteresis loop classified as type IV isotherm, indicating the porous silica shell. The BET specific surface area, pore volume and pore size of SNT- $40(40 \mathrm{~nm} \times 25 \mathrm{~nm})$ were calculated as $259 \mathrm{~m}^{2} \mathrm{~g}^{-1}, 0.676 \mathrm{~cm}^{3} \mathrm{~g}^{-1}$ and $2.4 \mathrm{~nm}$, respectively. The BET surface areas, pore volumes and pore sizes of SNT55 and SNT-150 were determined as $118 \mathrm{~m}^{2} \mathrm{~g}^{-1}$, $0.457 \mathrm{~cm}^{3} \mathrm{~g}^{-1}, \quad 3.2 \mathrm{~nm}$ and $119 \mathrm{~m}^{2} \mathrm{~g}^{-1}, \quad 0.311 \mathrm{~cm}^{3} \mathrm{~g}^{-1}$, $3.1 \mathrm{~nm}$, respectively. The porous shell and hollow inner space of these nanotubes make them potential candidates for drug delivery systems.

After modification with LA and adsorption of the CuS NPs, black dots with a size of about $5 \mathrm{~nm}$ can be clearly seen on the outer side of the SNTs (Fig. 1b). The SNTs with different lengths can be functionalized with CuS NPs through the same method (Figs S1, S2). Both of the UV-

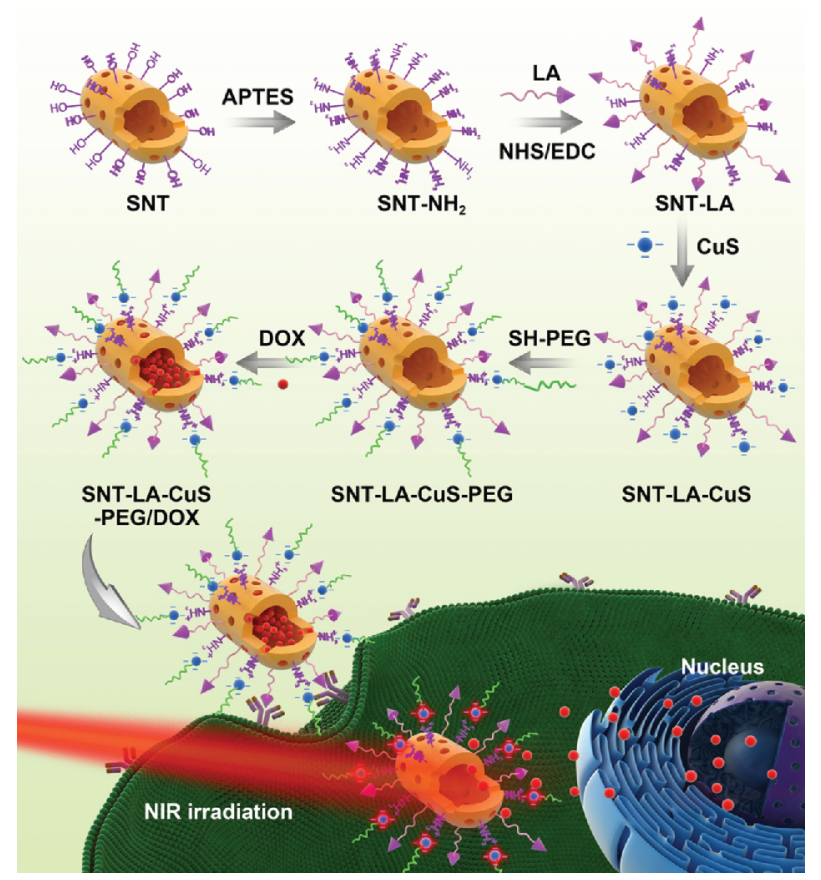

Scheme 1 The synthesis process of the nanocomposite and its application for chemo-photothermal therapy.
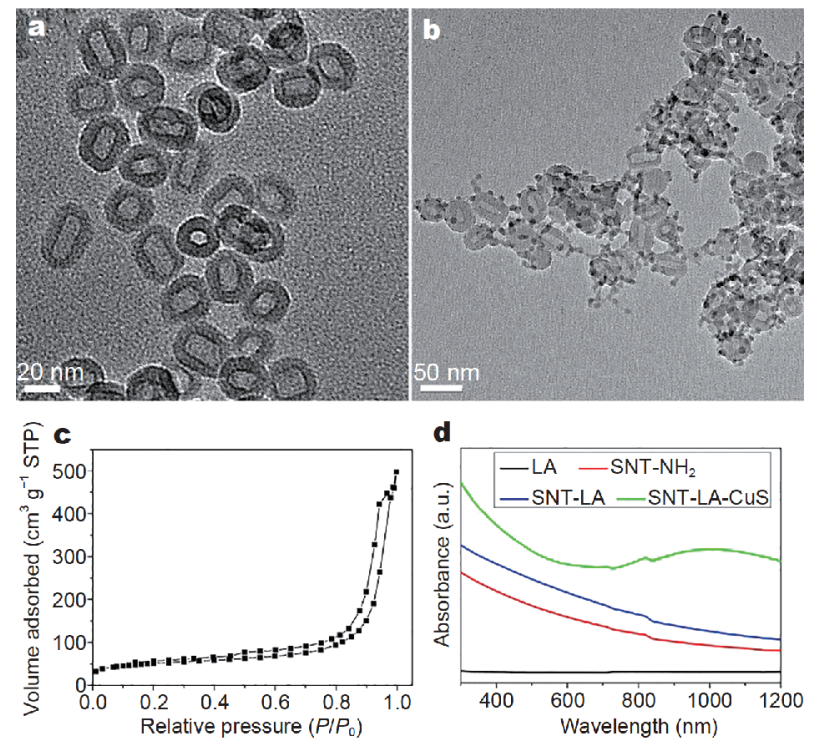

Figure 1 TEM images of SNT-40 (a) and SNT-40-LA-CuS-PEG (b); (c) $\mathrm{N}_{2}$ adsorption/desorption curve of SNT-40; (d) UV-vis-NIR spectra of the corresponding products.

vis-NIR absorption spectra of SNT-40- $\mathrm{NH}_{2}$ and SNT-40LA display no absorption peak from 800 to $1200 \mathrm{~nm}$ (Fig. 1d). SNT-40-LA-CuS-PEG nanocomposites show obvious absorption in the NIR region owing to the at- 
tached CuS NPs, which is consistent with the previous reports and guarantees that the NPs can be used for PTT [15].

The XRD pattern of the SNT-40-LA-CuS-PEG is shown in Fig. 2 . The broad band around the $2 \theta=22^{\circ}$ corresponds well with the characteristic reflection from amorphous $\mathrm{SiO}_{2}$ (JCPDS 29-0085). The peak at $47.9^{\circ}$ of the sample could be indexed to the (110) plane of CuS (JCPDS NO 06-0464). The broadened feature of the XRD patterns derives from the small size of CuS particles [37]. The XPS results shown in Fig. 3 further identify the composition of the sample. Except for Si $2 p$ and $\mathrm{O} 1 \mathrm{~s}, \mathrm{Cu} 2 \mathrm{p}$ spectrum of SNT-40-LA-CuS at 932.6 and $952.2 \mathrm{eV}$ are indexed to $\mathrm{Cu} 2 \mathrm{p}_{3 / 2}$ and $\mathrm{Cu} 2 \mathrm{p}_{1 / 2}$, which can be ascribed to binding energies for the $\mathrm{Cu} 2 \mathrm{p}$ orbital in accord with $\mathrm{Cu}(\mathrm{II})$ [38]. The $\mathrm{S} 2 \mathrm{p}$ spectrum further confirms the existence of $\mathrm{CuS}$. The peaks of $\mathrm{S} 2 \mathrm{p}_{3 / 2}$ and $\mathrm{S} 2 \mathrm{p}_{1 / 2}$ of the SNT-40-LA-CuS located at 162.4 and $168.2 \mathrm{eV}$ match well with the previously reported value [39].

The FT-IR spectra of SNT-40, SNT-40-NH ${ }_{2}$, SNT-40LA and LA are displayed in Fig. S4. SNT-40 exhibits peaks at $3419 \mathrm{~cm}^{-1}$ (O-H stretching), $1635 \mathrm{~cm}^{-1}$ (bending of adsorbed water), $1089 \mathrm{~cm}^{-1}$ (Si-O asymmetrical stretching), $950 \mathrm{~cm}^{-1}$ (Si-OH bending), and $800 \mathrm{~cm}^{-1}$ (inplane bending of geminal silanol). The peak around $1475 \mathrm{~cm}^{-1}$ after the reaction of silica NPs with APTES indicates the amino groups, demonstrating the formation of SNT-40-NH $\mathrm{N}_{2}$. The band between 3400 and $2400 \mathrm{~cm}^{-1}$ in the spectrum of pure LA is derived from $-\mathrm{OH}$ absorption. The characteristic band at $1740 \mathrm{~cm}^{-1}$ can be ascribed to the carbonyl stretching $(\mathrm{C}=\mathrm{O})$ of carboxylic groups. After the formation of the amide bond between carboxylic groups of LA and amine groups of SNT-40$\mathrm{NH}_{2}$, the carbonyl stretching of LA is absent [40]. The peaks of 1557,1438 and $1418 \mathrm{~cm}^{-1}$ observed in the spectrum of SNT-40-LA indicate the successful grafting of LA molecules.

Zeta potentials of SNT-40, SNT-40-NH $\mathrm{NH}_{2}$, SNT-40-LA, SNT-40-LA-CuS, and SNT-40-LA-CuS-PEG were measured to evaluate the change of the surface charge of these samples. The zeta potential of SNT-40 is $-10.2 \mathrm{mV}$, confirming $-\mathrm{OH}$ groups on the surface of the pure silica. The zeta potential of SNT-40- $\mathrm{NH}_{2}$ is $17.0 \mathrm{mV}$, indicating the successful functionalization with the $\mathrm{NH}_{2}$ groups. After grafting with LA, the zeta potential of the composites decreases to $11.5 \mathrm{mV}$, which is mainly attributed to the partially shielding of $\mathrm{NH}_{2}$ groups by LA, demonstrating that LA is successfully grafted onto the SNT- $\mathrm{NH}_{2}$. The zeta potential of SNT-40-LA-CuS decreases to -3.96 $\mathrm{mV}$, which shows the successful coating of the CuS NPs

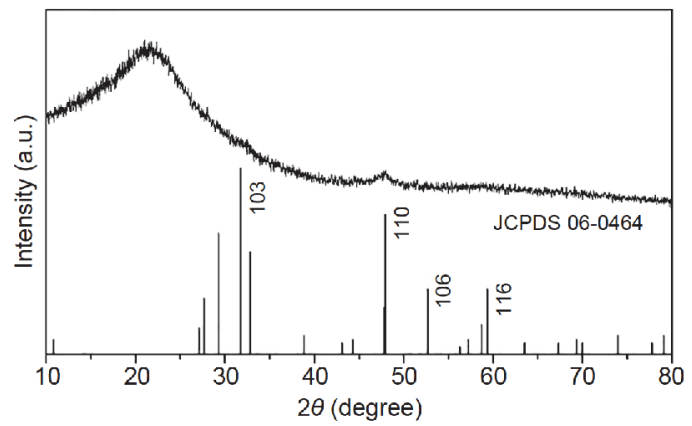

Figure 2 XRD pattern of the SNT-40-LA-CuS-PEG and the standard JCPDS card 06-0464 of CuS.
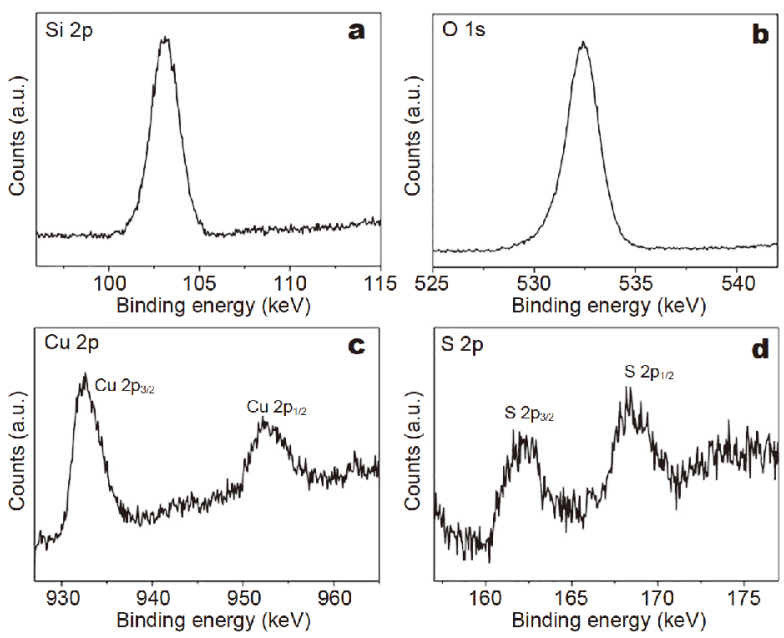

Figure 3 XPS spectra of the SNT-40-LA-CuS: (a) Si 2p, (b) O 1s, (c) $\mathrm{Cu} 2 \mathrm{p}$, and (d) S $2 \mathrm{p}$.

onto the SNT-LA. Furthermore, the long chain of PEG introduced may shield part charge, which leads to the decreasing potential of SNT-40-LA-CuS-PEG $(-2.32 \mathrm{mV})$.

A standard MTT assay was carried out to assess the cytotoxic effect of the nanocomposites. After incubation with the NPs at concentrations ranging from 6.25 to $200 \mu \mathrm{g} \mathrm{mL}^{-1}$ for $24 \mathrm{~h}$, the cell viability of L929 shows higher than $80 \%$ (Fig. S5). It can be demonstrated that the nanotubes with different lengths have good biocompatibility for potential applications in biomedical area.

The cellular endocytosis of SNT-40-- $\mathrm{NH}_{2}$-CuS-PEG and SNT-40-LA-CuS-PEG NPs by HepG2 cells was measured by ICP-OES. As shown in Table S1, the cell endocytosis of $\mathrm{Si}$ inside cells is $17.55,26.69,34.68 \mathrm{pg}$ per cell for SNT-40$\mathrm{NH}_{2}$-CuS-PEG at 1, 4 and $6 \mathrm{~h}$, respectively. Because the targeting of LA to receptor of the HepG2 cells, the total amounts of the Si for SNT-40-LA-CuS-PEG are more 
than twice that of SNT-40-NH $\mathrm{N}_{2}$-CuS-PEG, which are $39.17,54.96,75.78$ pg per cell. HepG2 cells were treated with LA-functionalized SNTs with various lengths on the basis of the same Si concentration. The Si contents inside the SNT-55-LA-CuS-PEG and SNT-150-LA-CuS-PEG are $34.97,43.35,56.40$ and $30.04,36.45,46.80$ pg per cell after incubation with HepG2 cells at 1,4 and $6 \mathrm{~h}$, respectively. With the increasing size of the SNTs, the content of $\mathrm{Si}$ in HepG2 cells decreases gradually.

To further study the effect of the targeted receptor on the interaction between the NPs and cancer cells, FITClabeled SNT-40- $\mathrm{NH}_{2}$-CuS-PEG and SNT-40-LA-CuSPEG were incubated with HepG2 cells for different time intervals, respectively. As shown in Fig. 4, gradually improved fluorescence intensity of FITC was detected with time increasing. This phenomenon suggests that the nanocomposites were gradually internalized into HepG2 cells with extension of time. Moreover, the SNT-40-LACuS-PEG shows much brighter green signal compared with SNT-40- $\mathrm{NH}_{2}$-CuS-PEG at the same incubation time. This phenomenon can be ascribed to the LA conjugated on the nanocomposites that can efficiently target the asialoglycoprotein receptor overexpressing on heptoma cells.

The fluorescence images of FITC for SNT-LA-CuSPEG (55 and $150 \mathrm{~nm}$ ) are also demonstrated in Fig. S6. The relatively lower fluorescence of FITC for SNT-150LA-CuS-PEG-FITC reveals that NPs with larger particle sizes have lower cell uptake capabilities. Based on these results, the SNTs with the length of $40 \mathrm{~nm}$ were chosen for further in vivo/vitro experiments.

The temperature of the solutions containing different concentrations of the NPs was recorded using the 808-nm laser irradiation $\left(2 \mathrm{~W} \mathrm{~cm}^{-2}\right)$ to investigate the photothermal effect. The pure water $\left(0 \mu \mathrm{g} \mathrm{mL}^{-1}\right)$ displayed a slight temperature increase (Fig. 5). The temperature of SNT-40 suspension with a concentration of $400 \mu \mathrm{g} \mathrm{mL}^{-1}$ showed a little elevation $\left(1.6^{\circ} \mathrm{C}\right)$ compared with the $\mathrm{CuS}$ loaded samples (Fig. S7). However, the temperature of the NPs aqueous solution showed a significant increase under the same condition. Moreover, the final temperature rose to $52.6^{\circ} \mathrm{C}$ when the $\mathrm{NP}$ concentration increased to $400 \mu \mathrm{g} \mathrm{mL} L^{-1}$.

Fig. 6 shows the infrared thermal images of a tumorbearing mouse. The images were collected by using an infrared camera at different time intervals under $808-\mathrm{nm}$ laser irradiation after the mouse was intravenously injected for $12 \mathrm{~h}$. The tumor exhibited a white color at the beginning, while became yellow then to red with increased irradiation time, and the temperature increased

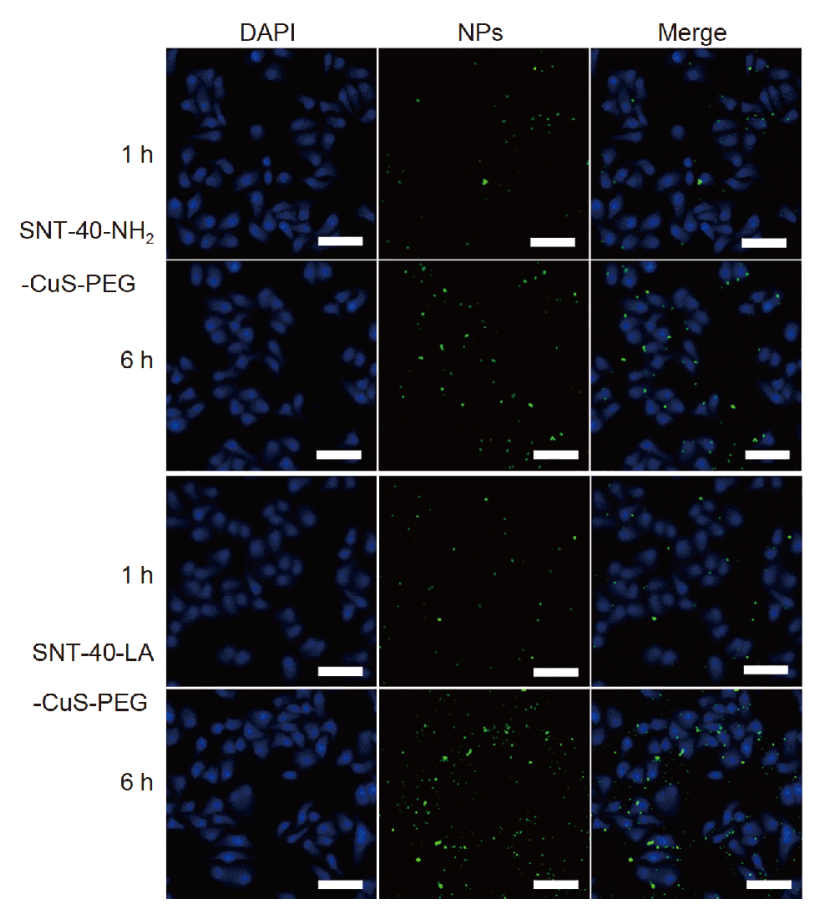

Figure 4 CLSM images for the cellular endocytosis of SNT-40- $\mathrm{NH}_{2}-$ CuS-PEG-FITC and SNT-40-LA-CuS-PEG-FITC after incubation with HepG2 cells for 1 and $6 \mathrm{~h}$, respectively. Scale bars represent $50 \mu \mathrm{m}$.

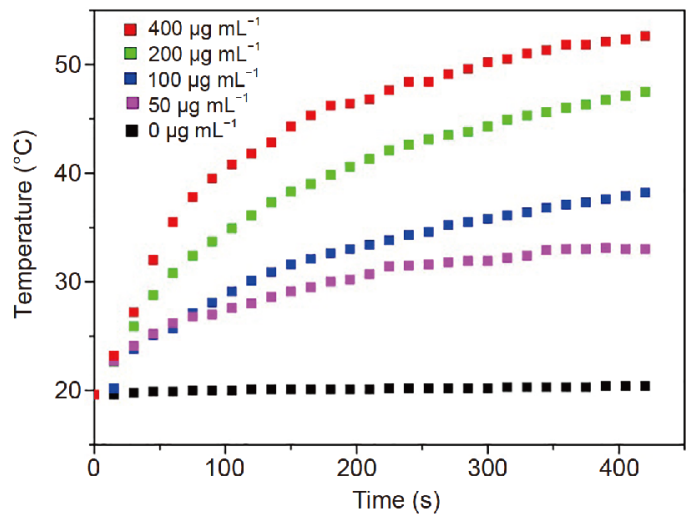

Figure 5 Temperature change curves of the solutions containing the SNT-40-LA-CuS-PEG with various concentrations under 808-nm NIR laser irradiation with power density of $2 \mathrm{~W} \mathrm{~cm}^{-2}$.

from 31.7 to $63.2^{\circ} \mathrm{C}$, while the temperature of the control group only reached to $38.6^{\circ} \mathrm{C}$. These images reveal that the nanocomposites can be effectively uptaken by tumor and convert near infrared light into heat because of the localized surface plasmon resonance of high-concentration free carrier (holes) due to $\mathrm{Cu}$ deficiency in the $\mathrm{CuS}$ NPs [21].

The loading amount ratio of DOX in the SNT-40-LACuS-PEG was determined to be $12 \%$. The release profiles 


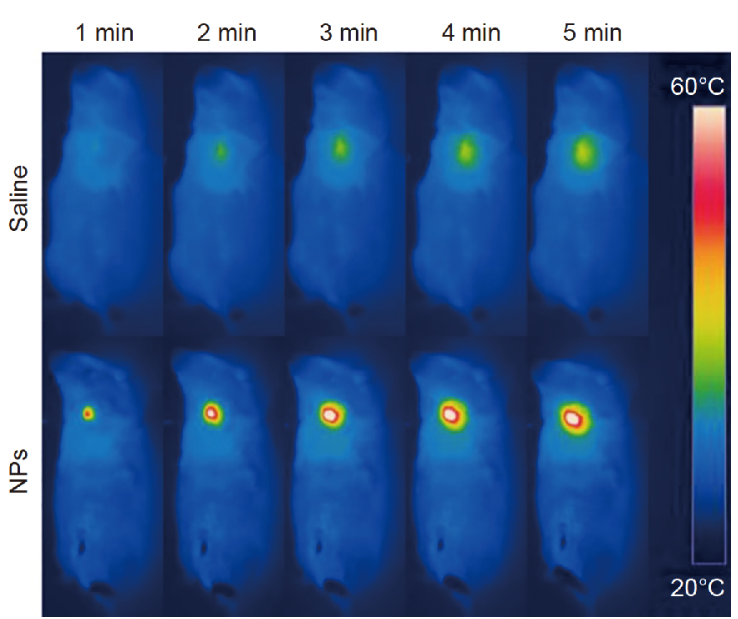

Figure 6 The infrared thermal images of tumor-bearing mice after injection of SNT-40-LA-CuS-PEG (NPs) correlated with the irradiation time exposed to $808-\mathrm{nm}$ NIR laser $\left(2 \mathrm{~W} \mathrm{~cm}^{-2}\right)$.

of DOX in buffer solutions with different $\mathrm{pH}$ values are displayed in Fig. 7. The DOX released from the SNT-40LA-CuS-PEG at pH 7.4 was only $12.98 \%$ in $8 \mathrm{~h}$, indicating that very few DOX molecules were released in the neutral solution. The accumulated release quantity of DOX reached $47 \%$ at $\mathrm{pH} 5.0$, which could be ascribed to the improved solubility of DOX in acidic solution and the weakened interaction between the DOX and silanol groups of the carrier. As shown, the release rate of DOX from the SNT-40-LA-CuS-PEG increases with decreasing $\mathrm{pH}$. The $\mathrm{pH}$-dependent release properties of DOX from the nanocomposites can be potentially used for cancer therapy. The photothermal effect generated by the $\mathrm{CuS}$ NPs on the release of DOX was investigated. During the release process, the NIR laser irradiation was carried out for 5 min every hour to assess the photothermal effect on the drug release. The release ratios of DOX before and after the NIR laser irradiation were monitored. The results displayed a triggered accelerating release of drug molecules upon NIR irradiation and slower release when the laser was absent. In acidic solution ( $\mathrm{pH}$ 5.0), the release quantity of DOX with NIR reached up to $76 \%$ within $8 \mathrm{~h}$, while only $47 \%$ DOX was accumulated without irradiation. At neutral $\mathrm{pH}$, the release rate was apparently slower than that at lower $\mathrm{pH} 5.0$, which showed $22 \%$ DOX released under NIR irradiation. Similarly, the release of DOX was also enhanced under laser. These values reveal that the release of DOX from the NPs could be triggered by $808-\mathrm{nm}$ NIR laser irradiation, owing to the photothermal effect of the $\mathrm{CuS}$ which promotes the drug release. The heat reduces the electrostatic interaction between DOX and the silanol groups on the silica tube,

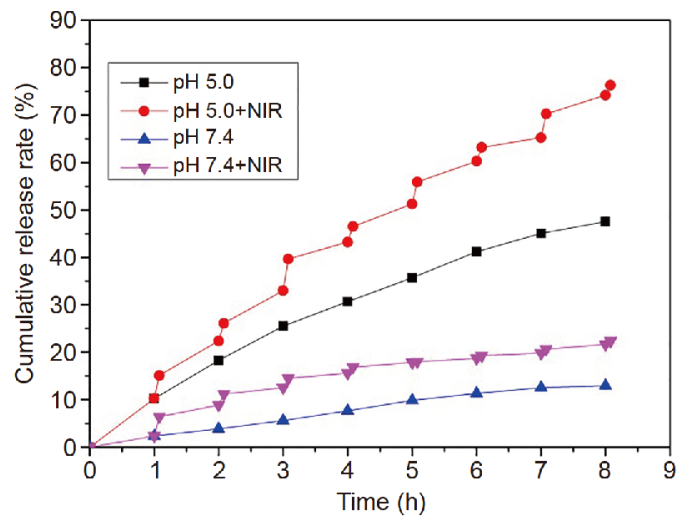

Figure 7 The release rate of DOX from the particles in PBS ( $\mathrm{pH} 7.4$ and $5.0)$ in presence or absence of 808-nm laser irradiation $\left(2 \mathrm{~W} \mathrm{~cm}^{-2}\right)$.

meanwhile between DOX and citrate, which leads to faster DOX release from the nanocomposites [26]. The $\mathrm{pH}$-dependent and NIR-triggered DOX release from the NPs can be applicable for the controllable drug release systems. The release of DOX is inhibited at the normal physiological environment, but the release could be accelerated after the nanocomposites are taken into acid cancer cell endosomes and lysosomes under NIR laser irradiation.

To test the targeting effect of the LA of the nanocomposites, we first incubated DOX loaded SNT-40$\mathrm{NH}_{2}$-CuS-PEG and SNT-40-LA-CuS-PEG with HepG2 cells for $24 \mathrm{~h}$. As revealed in Figs S8 and S9, pure SNT-40$\mathrm{NH}_{2}$-CuS-PEG, SNT-55-LA-CuS-PEG and SNT-150-LACuS-PEG show low cytotoxicity to HepG2 cells. Free DOX, DOX loaded SNT-40-NH $\mathrm{N}_{2}$-CuS-PEG and SNT-40LA-CuS-PEG exhibit notable cytotoxicity to HepG2 cells, and the inhibition ratio against HepG2 is elevated with higher concentration. The asialoglycoprotein receptor on the membrane of HepG2 cells could identify the LA group grafted on the DOX loaded SNT-40-LA-CuS-PEG, thus lead to higher internalization efficiency of the NPs and diffusion of the DOX inside cells. These results imply that SNT-40-LA-CuS-PEG/DOX could kill more cancer cells than SNT-40- $\mathrm{NH}_{2}$-CuS-PEG/DOX under the same conditions in vitro. Therefore, the NPs functionalized with LA can effectively be internalized by cancer cells with the receptor and the ablation of cancer cells can be promoted. Five groups of pure SNT-40-LA-CuS-PEG (NPs), NPs+NIR, DOX, NPs/DOX, NPs/DOX+NIR were used for incubation with HepG2 cells in order to further investigate the combination of chemo-therapy and PTT of the prepared NPs. As depicted in Fig. 8, the HepG2 cell viability after incubation with pure NPs is higher than 
$90 \%$, implying that the pure material is low toxic to cancer cells. The results suggest that the group of NPs/ DOX+NIR has the maximum inhibiting ability with the cell viability decreasing to $25 \%$. The increased cell inhibiting ability suggests that the synergistic effect of the chemo-photothermal system is greater than the individual chemical therapy or PTT. This can be attributed to the increased temperature-accelerated drug release and

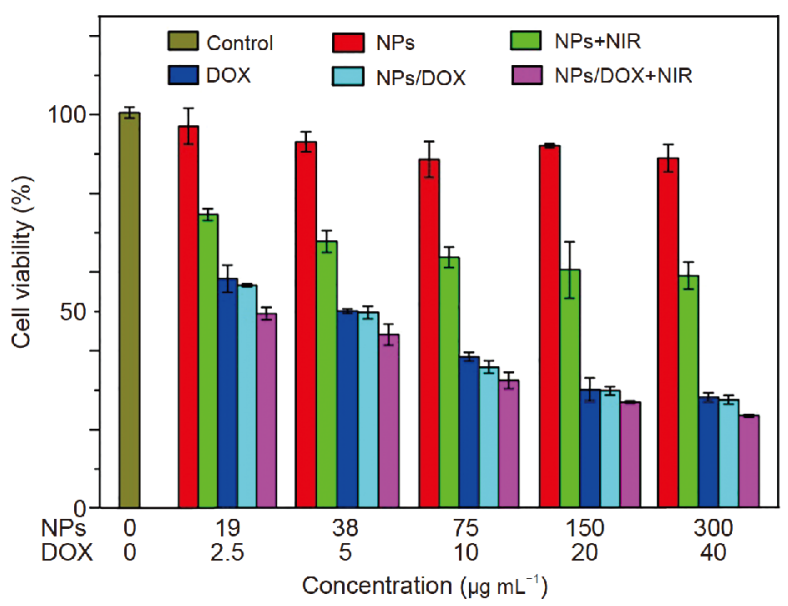

Figure 8 In vitro cell viability of HepG2 cells incubated with pure NPs, DOX, and NPs/DOX at different concentrations in presence or absence of NIR-laser irradiation for $24 \mathrm{~h}$. the thermal ablation of cancer cells.

The synergistic therapy effect of NPs was further determined in mice. DOX-NPs were injected intratumorally to the $\mathrm{H} 22$ tumor-bearing mice when the tumor size reached to about $100 \mathrm{~mm}^{3}$. The mice were sacrificed after treatment for 14 days. Fig. 9a shows the representative images of the excised tumors with different treatment
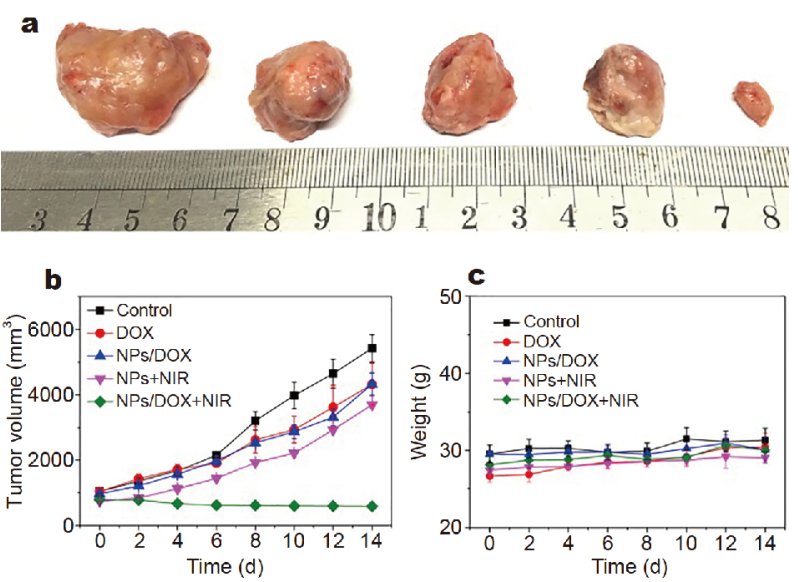

Figure 9 (a) Photographs of tumors from five groups of tumor-bearing mice (control, DOX, NPs/DOX, NPs+NIR, NPs/DOX+NIR). (b) The tumor volume of the mice of the corresponding groups versus the treatment time. (c) The body weight of the mice in different groups versus the treatment time.

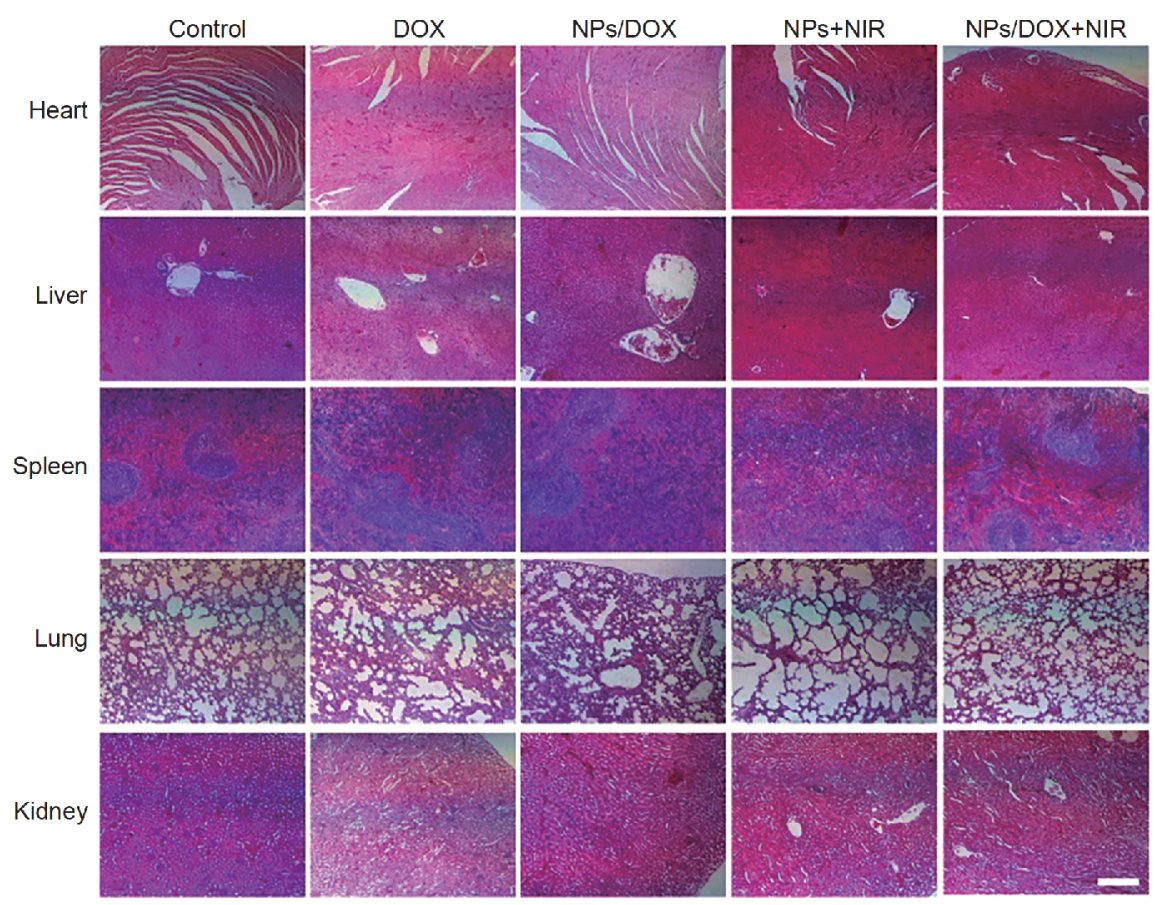

Figure 10 Representative histological H\&E stained tissue images of the major organs from mice at 14 days after treatment. The scale bar of all the pictures is $50 \mu \mathrm{m}$. 
conditions (with and without 808-nm laser irradiation). The tumor growth curves (Fig. 9b) indicate that the tumor size of the NPs/DOX under irradiation group decrease obviously in comparison with other groups, which is consistent with the graphs of the excised tumor. As shown in Fig. 9c, the weight changes of the mice are not obvious during the treatment. These results imply that the integration of the chemo-photothermal therapy exhibits considerably higher tumor growth inhibition.

$\mathrm{H} \& \mathrm{E}$ staining analyses of the main organs of the mice, including heart, liver, spleen, lung, and kidney in different groups were also performed after the various therapy treatments. No obvious pathological changes were observed in the main organs, confirming the good biocompatibility of NPs (Fig. 10).

\section{CONCLUSION}

Hollow structured SNTs with controllable size decorated with heat generating agent $\mathrm{CuS}$ NPs and target LA group were prepared. The SNTs with average size of $40 \mathrm{~nm} \times 25 \mathrm{~nm}$ show the highest cell uptake amount after incubation with HepG2 cells. CuS NPs adsorbed onto the SNTs can effectively convert NIR light to heat upon the 808-nm NIR irradiation. The introduced target groups efficiently enhance the internalization of the NPs. The synthesized nanocomposites have good biocompatibility and photothermal property. The DOX-loaded nanocomposites show $\mathrm{pH}$-dependent and NIR-triggered drug release, which causes much higher ablation of cancer cells under synergistic therapy. The in vivo cell cultivation and histological section analyses reveal that the DOX-loaded NPs can reduce the tumor growth, and cause no obvious damage to the healthy organs. The chemo-photothermal therapy vehicles with target ligand achieved a higher therapeutic efficacy than that of individual chemotherapy or photothermal therapy. Hence, the SNT-based nanoplatform could be potentially used in biomedical applications.

Received 4 November 2019; accepted 18 December 2019; published online 15 January 2020

1 He Q, Shi J. MSN anti-cancer nanomedicines: chemotherapy enhancement, overcoming of drug resistance, and metastasis inhibition. Adv Mater, 2014, 26: 391-411

2 Pan L, He Q, Liu J, et al. Nuclear-targeted drug delivery of TAT peptide-conjugated monodisperse mesoporous silica nanoparticles. J Am Chem Soc, 2012, 134: 5722-5725

3 Lee SB, Kim HL, Jeong HJ, et al. Mesoporous silica nanoparticle pretargeting for PET imaging based on a rapid bioorthogonal reaction in a living body. Angew Chem Int Ed, 2013, 52: 1054910552
4 Cheng L, Yang L, Meng F, Zhong Z. Protein nanotherapeutics as an emerging modality for cancer therapy. Adv Healthcare Mater, 2018, 7: 1800685

5 Yang B, Chen Y, Shi J. Mesoporous silica/organosilica nanoparticles: Synthesis, biological effect and biomedical application. Mater Sci Eng-R-Rep, 2019, 137: 66-105

6 Zhao W, Gu J, Zhang L, Chen H, Shi J. Fabrication of uniform magnetic nanocomposite spheres with a magnetic core/mesoporous silica shell structure. J Am Chem Soc, 2005, 127: 8916-8917

7 Chen Y, Chen H, Zeng D, et al. Core/shell structured hollow mesoporous nanocapsules: a potential platform for simultaneous cell imaging and anticancer drug delivery. ACS Nano, 2010, 4: 6001-6013

8 Hao N, Li L, Zhang Q, et al. The shape effect of PEGylated mesoporous silica nanoparticles on cellular uptake pathway in hela cells. Microporous Mesoporous Mater, 2012, 162: 14-23

9 Huang X, Teng X, Chen D, Tang F, He J. The effect of the shape of mesoporous silica nanoparticles on cellular uptake and cell function. Biomaterials, 2010, 31: 438-448

10 Decuzzi P, Godin B, Tanaka T, et al. Size and shape effects in the biodistribution of intravascularly injected particles. J Control Release, 2010, 141: 320-327

11 Gao C, Zhang Q, Lu Z, Yin Y. Templated synthesis of metal nanorods in silica nanotubes. J Am Chem Soc, 2011, 133: 1970619709

12 Gao C, Lu Z, Yin Y. Gram-scale synthesis of silica nanotubes with controlled aspect ratios by templating of nickel-hydrazine complex nanorods. Langmuir, 2011, 27: 12201-12208

13 Huang L, Ao L, Wang W, et al. Multifunctional magnetic silica nanotubes for MR imaging and targeted drug delivery. Chem Commun, 2015, 51: 3923-3926

14 Yang X, He D, He X, et al. Synthesis of hollow mesoporous silica nanorods with controllable aspect ratios for intracellular triggered drug release in cancer cells. ACS Appl Mater Interfaces, 2016, 8: 20558-20569

15 Deng X, Li K, Cai X, et al. A hollow-structured CuS@ $\mathrm{Cu}_{2} \mathrm{~S} @ \mathrm{Au}$ nanohybrid: synergistically enhanced photothermal efficiency and photoswitchable targeting effect for cancer theranostics. Adv Mater, 2017, 29: 1701266

16 Li Y, Bai G, Zeng S, Hao J. Theranostic carbon dots with innovative NIR-II emission for in vivo renal-excreted optical imaging and photothermal therapy. ACS Appl Mater Interfaces, 2019, 11: 4737-4744

17 Zhou M, Zhang R, Huang M, et al. A chelator-free multifunctional $\left[{ }^{64} \mathrm{Cu}\right] \mathrm{CuS}$ nanoparticle platform for simultaneous micro-PET/CT imaging and photothermal ablation therapy. J Am Chem Soc, 2010, 132: $15351-15358$

18 Guo L, Panderi I, Yan DD, et al. A comparative study of hollow copper sulfide nanoparticles and hollow gold nanospheres on degradability and toxicity. ACS Nano, 2013, 7: 8780-8793

19 Xiao Q, Zheng X, Bu W, et al. A core/satellite multifunctional nanotheranostic for in vivo imaging and tumor eradication by radiation/photothermal synergistic therapy. J Am Chem Soc, 2013, 135: 13041-13048

20 Zha Z, Wang S, Zhang S, et al. Targeted delivery of CuS nanoparticles through ultrasound image-guided microbubble destruction for efficient photothermal therapy. Nanoscale, 2013, 5: 32163219

21 Liang S, Deng X, Chang Y, et al. Intelligent hollow Pt-CuS janus architecture for synergistic catalysis-enhanced sonodynamic and 
photothermal cancer therapy. Nano Lett, 2019, 19: 4134-4145

22 Zhang Y, Hou Z, Ge Y, et al. DNA-hybrid-gated photothermal mesoporous silica nanoparticles for NIR-responsive and aptamertargeted drug delivery. ACS Appl Mater Interfaces, 2015, 7: 2069620706

23 Chen Y, Hou Z, Liu B, et al. DOX-Cu $\mathrm{S}_{5} @ \mathrm{mSiO}_{2}-\mathrm{PG}$ composite fibers for orthotopic synergistic chemo- and photothermal tumor therapy. Dalton Trans, 2015, 44: 3118-3127

24 Jiang M, Liu H, Zeng S, Hao J. A general in situ growth strategy of designing theranostic $\mathrm{NaLnF}_{4} @ \mathrm{Cu}_{2-x} \mathrm{~S}$ nanoplatform for in vivo NIR-II optical imaging beyond $1500 \mathrm{~nm}$ and photothermal therapy. Adv Therap, 2019, 2: 1800153

25 Li Y, Zeng S, Hao J. Non-invasive optical guided tumor metastasis/ vessel imaging by using lanthanide nanoprobe with enhanced down-shifting emission beyond $1500 \mathrm{~nm}$. ACS Nano, 2019, 13: 248-259

$26 \mathrm{Wu} \mathrm{L}, \mathrm{Wu} \mathrm{M}$, Zeng $\mathrm{Y}$, et al. Multifunctional PEG modified DOX loaded mesoporous silica nanoparticle@CuS nanohybrids as photo-thermal agent and thermal-triggered drug release vehicle for hepatocellular carcinoma treatment. Nanotechnology, 2015, 26: 025102

27 Shi S, Chen F, Cai W. Biomedical applications of functionalized hollow mesoporous silica nanoparticles: focusing on molecular imaging. Nanomedicine, 2013, 8: 2027-2039

28 Wang X, Li X, Ito A, et al. Stimulation of in vivo antitumor immunity with hollow mesoporous silica nanospheres. Angew Chem Int Ed, 2016, 55: 1899-1903

29 Liu X, Fu F, Xu K, et al. Folic acid-conjugated hollow mesoporous silica/CuS nanocomposites as a difunctional nanoplatform for targeted chemo-photothermal therapy of cancer cells. J Mater Chem B, 2014, 2: 5358

30 Xie X, Gao N, Deng R, et al. Mechanistic investigation of photon upconversion in $\mathrm{Nd}^{3+}$-sensitized core-shell nanoparticles. J Am Chem Soc, 2013, 135: 12608-12611

31 Shen J, Chen G, Vu AM, et al. Engineering the upconversion nanoparticle excitation wavelength: cascade sensitization of tri-doped upconversion colloidal nanoparticles at $800 \mathrm{~nm}$. Adv Opt Mater, 2013, 1: 644-650

32 Sun LD, Wang YF, Yan CH. Paradigms and challenges for bioapplication of rare earth upconversion luminescent nanoparticles: small size and tunable emission/excitation spectra. Acc Chem Res, 2014, 47: 1001-1009

33 Jayakumar MKG, Idris NM, Huang K, Zhang Y. A paradigm shift in the excitation wavelength of upconversion nanoparticles. Nanoscale, 2014, 6: 8441-8443

34 Duan X, Li Y. Physicochemical characteristics of nanoparticles affect circulation, biodistribution, cellular internalization, and trafficking. Small, 2013, 9: 1521-1532
35 Yang R, Meng F, Ma S, et al. Galactose-decorated cross-linked biodegradable poly(ethylene glycol)- $b$-poly( $\varepsilon$-caprolactone) block copolymer micelles for enhanced hepatoma-targeting delivery of paclitaxel. Biomacromolecules, 2011, 12: 3047-3055

36 Sun L, Zhang X, An J, et al. Boronate ester bond-based core-shell nanocarriers with $\mathrm{pH}$ response for anticancer drug delivery. RSC Adv, 2014, 4: 20208-20215

37 Bi H, Dai Y, Lv R, et al. Doxorubicin-conjugated CuS nanoparticles for efficient synergistic therapy triggered by near-infrared light. Dalton Trans, 2016, 45: 5101-5110

38 Guo Z, Seol ML, Kim MS, et al. Hollow CuO nanospheres uniformly anchored on porous Si nanowires: preparation and their potential use as electrochemical sensors. Nanoscale, 2012, 4: 75257531

39 Liu P, Huang Y, Yan J, Yang Y, Zhao Y. Construction of CuS nanoflakes vertically aligned on magnetically decorated graphene and their enhanced microwave absorption properties. ACS Appl Mater Interfaces, 2016, 8: 5536-5546

40 Zhao R, Li T, Zheng G, et al. Simultaneous inhibition of growth and metastasis of hepatocellular carcinoma by co-delivery of ursolic acid and sorafenib using lactobionic acid modified and $\mathrm{pH}$ sensitive chitosan-conjugated mesoporous silica nanocomplex. Biomaterials, 2017, 143: 1-16

Acknowledgements This work was financially supported by the National Natural Science Foundation of China (NSFC, 51572257, 51720105015, 51672269, 51929201, 51922097, 51772124 and 51872282), the Science and Technology Cooperation Project between Chinese and Australian Governments (2017YFE0132300), the Key Research Program of Frontier Sciences, CAS (YZDY-SSW-JSC018), the Overseas, Hong Kong \& Macao Scholars Collaborated Researching Fund (21728101), the CAS-Croucher Funding Scheme for Joint Laboratories (CAS18204), Jiangmen Innovative Research Team Program (2017), and the Major Program of Basic Research and Applied Research of Guangdong Province (2017KZDXM083)

Author contributions Huang $S$ performed the experiments and wrote the draft of manuscript. Liu B, Deng X and Xie Z helped design the cell and animal experiments. Wei Y contributed to the CLSM image and analysis. Ma P, Cheng $\mathrm{Z}$ and Xing B provided suggestions and comments on the manuscript. Lin J proposed the project and revised the manuscript.

Conflict of interest The authors declare that they have no conflict of interest.

Supplementary information online version of the paper.
Supporting data are available in the 


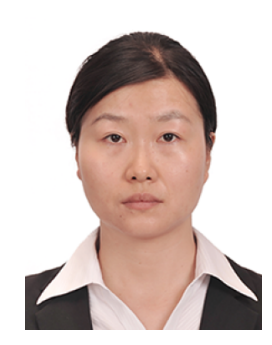

Shanshan Huang received her $\mathrm{PhD}$ degree in inorganic chemistry from the University of Chinese Academy of Sciences in 2013. She worked in Changchun Institute of Applied Chemistry (CIAC), Chinese Academy of Sciences (CAS) from 2006 to 2018. Currently, she is an associate professor in the College of Chemistry and Pharmaceutical Engineering, Huanghuai University. Her research interests include multifunctional mesoporous silica materials for drug delivery systems and rare-earth doped luminescent materials.

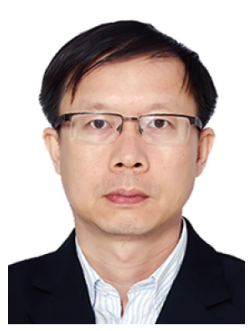

Ziyong Cheng received his BSc degree in materials engineering from Changchun University of Technology in 1994 and his $\mathrm{PhD}$ degree from CIAC, CAS in 2006. Following postdoctoral studies at Max-Planck Institute for Polymer Research (Mainz, Germany), he returned to CIAC (2008) to take up an associate professor position in inorganic chemistry. In 2013, he was promoted to a full professor. His research interests are nanostructured materials including perovskite quantum dots in photoelectric applications and polymer-inorganic nanocomposites for biomaterial related fields.

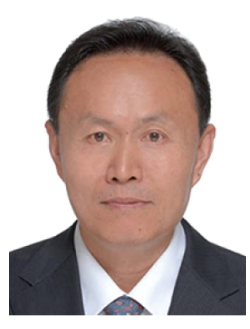

Jun Lin received his BSc and MSc degrees in inorganic chemistry from Jilin University in 1989 and 1992, respectively, and a $\mathrm{PhD}$ degree (inorganic chemistry) from CIAC, CAS in 1995. He worked as a postdoctoral researcher for more than 4 the years in City University of Hong Kong (1996), Institute of New Materials (Germany, 1997), Virginia Commonwealth University (USA, 1998), and University of New Orleans (USA, 1999). He is a professor at the CIAC, CAS. His research interests include luminescent materials and multifunctional composite materials toward their application in display, lighting and biomedical fields.
可控合成空心多孔氧化硅纳米管/CuS体系应用于 化疗-光热靶向治疗

${\text { 黄珊珊 }{ }^{1 *} \text {, 马平安 }{ }^{2}, \text { 韦祎 }^{2} \text {, 程子泳 } 2^{2 *} \text {, 刘蓓 }}^{2}$, 邓晓然 ${ }^{2}$, 谢忠曦 ${ }^{2}$, 邢本刚 ${ }^{4}$, 林君 ${ }^{2,3 *}$

摘要 多功能药物载体的设计合成并应用于肿瘤的联合治疗得到 了研究人员的广泛关注. 本文介绍了一种连接靶向基团的化疗-光 热联合治疗纳米平台. 首先制备了尺寸可控的平均长度为 $40 、 55$ 和150 $\mathrm{nm}$ 的空心多孔氧化硅纳米管, 在表面修饰具有光热功能的 硫化铜纳米颗粒, 然后连接乳糖酸基团实现肝癌细胞靶向功能. 平 均长度为 $40 \mathrm{~nm}$ 、修饰靶向基团的空心多孔材料显示出良好的生 物相容性, 且具有最大的HepG2细胞吞噬量. 负载盐酸阿霉素的纳 米复合材料表现出 $\mathrm{pH}$ 和 $808 \mathrm{~nm}$ 近红外激光刺激响应的释放效果. 将 $\mathrm{CuS}$ 光热治疗和盐酸阿霉素化疗相结合的方法在体外和体内的 抑制肿瘤效果都优于单独治疗. 研究结果表明, 该纳米复合材料在 化疗-光热联合治疗方面具有潜在的应用价值. 